\title{
ABSOLUTE PROPER MOTIONS OF BULGE GIANTS IN THE HIPPARCOS SYSTEM
}

\author{
D. MINNITI ${ }^{1}$, M.G.LATTANZI ${ }^{2}$, J.J. CLARIA ${ }^{3}$, G. MASSONE ${ }^{2}$, R. CASALEGNO ${ }^{2}$ \\ ${ }^{1}$ Lawrence Livermore National Laboratory, Livermore, CA, USA \\ 2 Osservatorio Astronomico Torino, Pino T.se, TO, ITALY \\ 3 Observatorio Astronomico, Cordoba, ARGENTINA
}

Three windows towards the Galactic bulge, located at $(l, b)=(1.6,-2.8),(5.2,-3.5)$, and $(4.2,-4.8)$, were identified from deep astrometric plates. Deep BVRI CCD photometry of these fields reaching $V=18.5$ was obtained at the ESO Dutch telescope. The color-color and color magnitude diagrams of these fields allow us to select different bulge tracers, including blue horizontal branch stars, red clump giants, and RGB tip stars, as well as disk main-sequence stars. A first catalogue of some 2000 red clump giants was constructed, and measurement of their proper motions in under way. We are deriving absolute proper motions in the Hipparcos system using a two step procedure. First, plates from the Cordoba archive of the Astrographic Catalogue (epochs 1903-1913) are used in combination with recent plates from the ESO GPO telescope to tie a set of intermediate magnitude field stars in the Hipparcos system of positions and proper motions. Second, deeper intermediate epoch (1958) and final epoch CCD images (epoch 1996.5) are used to determine the absolute proper motions of the fainter bulge stars. The average uncertainty in the absolute proper motion of a single giant, based on a preliminary sample of few tens of stars, is $0 " .006 \mathrm{yr}^{-1}$. This relatively small error, in combination with the sample size, would allow us to study the shape of the velocity ellipsoid for the different Galactic components present in the inner regions as function of Galactocentric distance, and also to measure an accurate distance to the Galactic center and the mass of the bulge. In addition, we identified about 20 faint objects which are potential members of the Sgr dwarf, based on their location in the color-magnitude diagrams, and are also measuring their proper motions.

Other windows with measured proper motions are located along the Galactic minor axis, including Baade's window at $l, b=(1,-4)$ (Spaenhauer, A., Jones, B. F., \& Whitford, A. E., 1992, $A J, 103,297$ ), and the Plaut field at $l, b=(0,-8)$ (Mendez, R. et al., 1997, in 4th ESO/CTIO Workshop on "The Galactic Center", in press). Our proper motions are complementary, probing the kinematics off the Galactic minor axis, where the signature of rotation should be evident. The fields chosen for this study overlap fields that the MACHO project is following since 1993. Their variable star database would provide with excellent tracers of different populations. The 3-D motions of these different Galactic components would be measured for the first time. These include RR Lyrae tracers of the inner metal-poor halo, Miras, LPVs and delta Scuti stars tracers of the metal-rich bulge, and eclipsing binaries tracers of the Galactic disk (Minniti, D., et al., 1996, in IAP Coll. on "Variable Stars and the Astrophysical Returns of Microlensing Surveys", ed. R. Ferlet, p. 257; Alcock, C., et al., 1997, astro-ph/9707311). 